\title{
A Study on Fluidic Diode for Wave Energy Conversion-Effect of Bypass Geometry on the Turbine Performance
}

\author{
Keito Matsumoto1, Manabu Takao', Shinya Okuhara1, Miah Md. Ashraful Alam¹, Yoichi Kinoue ${ }^{2}$ \\ ${ }^{1}$ National Institute of Technology, Matsue College, Matsue, Japan \\ ${ }^{2}$ Institute of Ocean Energy, Saga University, Saga, Japan \\ Email: takao@matsue-ct.jp
}

How to cite this paper: Matsumoto, K., Takao, M., Okuhara, S., Alam, M.M.A. and Kinoue, Y. (2020) A Study on Fluidic Diode for Wave Energy Conversion-Effect of Bypass Geometry on the Turbine Performance. Open Journal of Fluid Dynamics, 10, 270-278. https://doi.org/10.4236/ojfd.2020.103016

Received: August 20, 2020

Accepted: September 21, 2020

Published: September 24, 2020

Copyright $\odot 2020$ by author(s) and Scientific Research Publishing Inc. This work is licensed under the Creative Commons Attribution International License (CC BY 4.0).

http://creativecommons.org/licenses/by/4.0/

\begin{abstract}
A twin-impulse turbine for bi-directional flow has been developed for wave energy converter. However, the previous studies elucidated that the mean efficiency of the twin turbine is much lower than that of the impulse turbine for a unidirectional flow because a portion of airflow passes through the reverse flow turbine whose efficiency is very low. Therefore, a fluidic diode was adopted in the twin-impulse turbine in order to reduce the air flow through the reverse flow turbine. In this study, the rectification effect of the fluidic diode was investigated where a bypass is introduced into a blunt body. A computational fluid dynamics (CFD) analysis was conducted to investigate the effect of fluidic diodes on the turbine performance. In this analysis, RANS equations were used as the governing equations and the standard $k-\mathcal{E}$ model was used as the turbulence model. The computational domain is composed of a circular tube and fluidic diode, and the domain meshed with an approximately 1.5 million mesh elements. As a result, it was found that the rectification effect of the fluidic diode is enhanced by installing a blunt body with a bypass hole of $5^{\circ}$ taper angle.
\end{abstract}

\section{Keywords}

Fluidic Diode, Wave Energy Conversion, Twin-Impulse Turbine, CFD Analysis

\section{Introduction}

Japan is surrounded by the ocean, and abundant wave energy exists around the country. Wave energy converter is one of the wave energy utilization techniques, and there are three types of converters, namely, movable body type, over topping 
type and oscillating water column type.

In this study, the oscillating water column (OWC) type wave energy converter was used because of its simple structure and the characteristic feature of the use in abnormal sea conditions. The OWC-based power generator converts the wave energy into the electrical energy driving an air turbine coupled with an electrical generator by means of the reciprocating air flow generated in the air chamber [1]. A twin-impulse turbine as shown in Figure 1 has been developed for the OWC-based wave energy converter. A more details of the turbine cascade can be found in Refs. [2] [3]. Since, an impulse turbine, which is used for unidirectional airflow, cannot obtain a high efficiency in a reverse flow. Thus, a fluidic diode has been used to alleviate this problem by rectifying the flow in the turbine [4]. However, the rectification effect of the current fluid diode is about $70 \%$, which is not feasible to use in a practical application, so far.

Therefore, in this study, the flow rectification performance of fluidic diode was investigated by a computational fluid dynamics (CFD) analysis. Specifically, in order to achieve a further improvement in the rectification characteristics, the rectification effect of the fluidic diode was investigated when a bypass, aimed at increasing the flow rate in the forward flow turbine, was provided into a blunt body.

\section{Fluidic Diode}

In the twin-impulse turbine, a fluidic diode is installed as shown in Figure 1 in order to reduce the amount of airflow into the turbine in reverse flow. The fluidic diode is expected to improve the average efficiency of the turbine by rectifying the reciprocating airflow and by increasing the flow rate in the forward flow turbine.

In previous studies, a fluidic diode with a cross-sectional shape shown in Figure 2(A) was adopted. This fluidic diode consists of a blunt body $(B)$ with a hollow $(\mathrm{H})$ at the back, a toroidal area $(T)$, and a nozzle $(N)$. The diameter of the larger part is $D=240 \mathrm{~mm}$. The airflow from the region $B$ to $N$ is called "forward flow", and a vice versa is called "reverse flow".

The previous studies shown that the rectifying characteristics are improved by

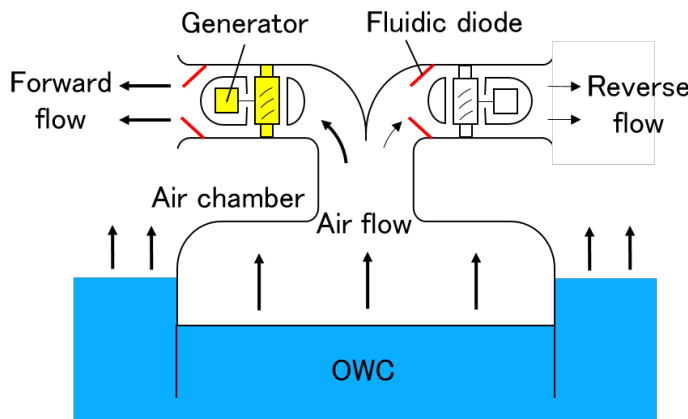

(A)

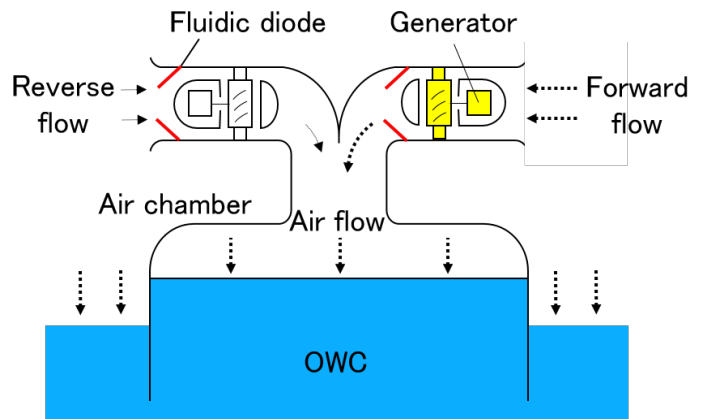

(B)

Figure 1. Twin-impulse turbine for wave energy conversion (A) Exhalation; (B) Inhalation. 


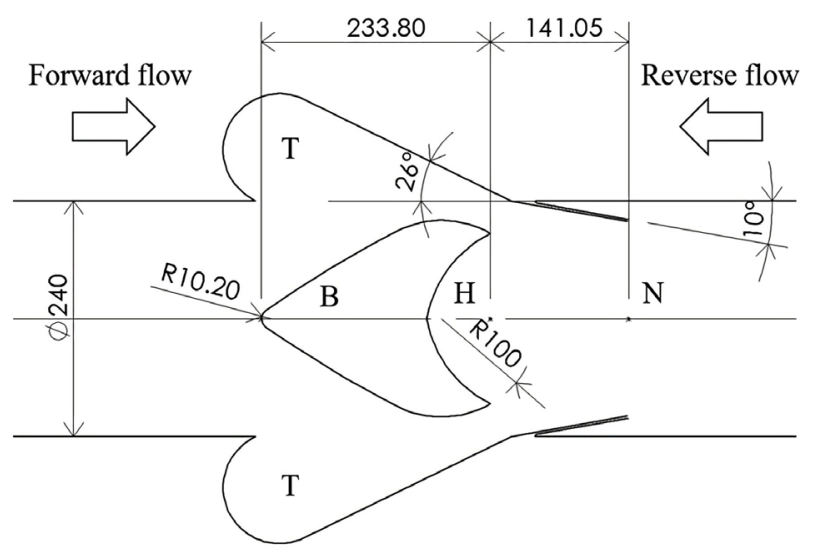

(A)

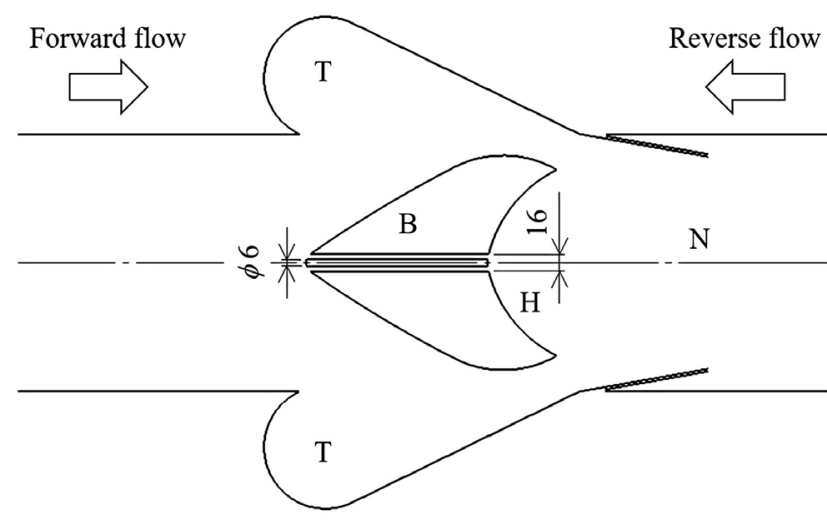

(C)

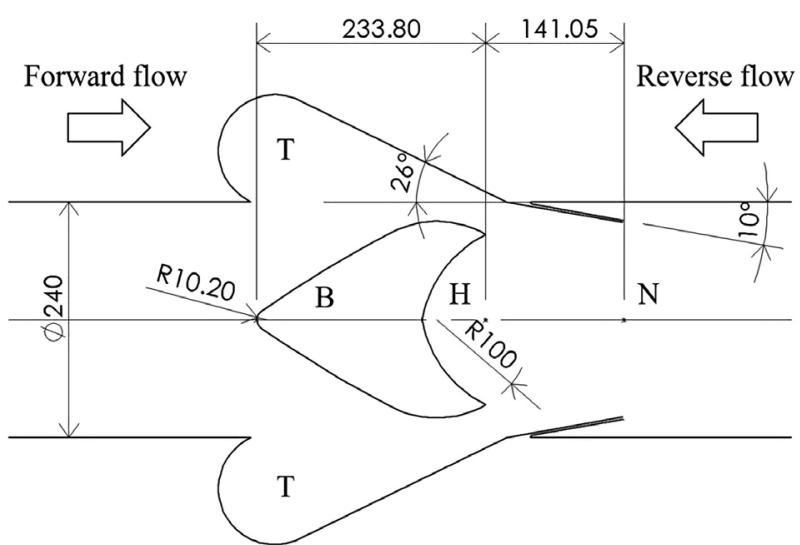

(B)

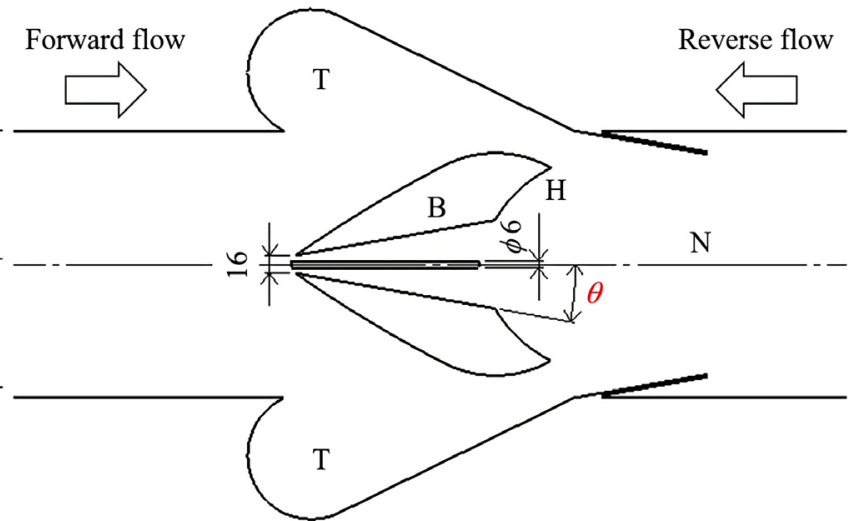

(D)

Figure 2. Tested fluidic diodes. (A) Original type; (B) Straight type; (C) Cylindrical type; (D) Taper cylindrical type.

providing a bypass on the blunt body in Figure 2(B). From a subsequent research, it was found that the bypass shape as shown in Figure 2(C), wherein a long and narrow rod was installed at the center of the bypass, can further improve the rectification characteristics.

In this study, in order to achieve further improvement in the rectification characteristics, a "taper cylindrical type" bypass as shown in Figure 2(D) was installed where the taper angle $\theta$ is ranging between $0^{\circ}$ and $15^{\circ}$.

\section{Methodologies}

The rectification effect of the fluidic diode has been investigated by using a computational fluid dynamics (CFD) analysis. The shape of the fluidic diode was varied as mentioned in the previous section, in order to investigate its effect. The CFD analysis was performed using a commercial software of SCRYU/Tetra by Cradle Co. Ltd. The RANS equations were used as the governing equations. The standard $k-\varepsilon$ model was used as the turbulence model. The computational domain of the flow path is composed of a circular tube and fluidic diode. The domain was meshed with approximately 1.5 million mesh elements. As the boun- 
dary conditions, the inner wall of the pipe and the fluidic diode were set to the no-slip conditions, to replicate the experimental results a constant flow rate of $Q$ $=0.256 \mathrm{~m}^{3} / \mathrm{s}$ was set at the inlet, and the outlet was open to the atmosphere. The pressure and velocity fields inside the fluidic diode were visualized and analyzed to evaluate the rectification effect. Since the fluidic diode is considered to have a higher rectification effect, the ratio of the pressure differences $\Delta p_{f}$ and $\Delta p_{r}$ across the diode generated in both forward and reverse flow turbines is higher, and the pressure ratio $R_{D}$ is defined as $R_{D}=\Delta p_{r} / \Delta p_{f}$

\section{Results and Discussion}

Figure 3 shows the effect of the taper angle of bypass hole on the ratio of pressure differences. It is found from the figure that the preferable angle is $\theta=5^{\circ}$ in the tested range. Therefore, this value of taper angle of bypass hole is adopted in this study.

Table 1 shows the effect of the bypass installed on the blunt body on the pressure difference $\Delta p_{f} \Delta p_{r}$ before and after the fluidic diode and on the pressure ratio $R_{D}$. The results of the diode with straight and cylindrical bypass obtained in the previous studies are also presented here for the comparison. The table shows that the bypass type diode has a higher pressure ratio $R_{D}$ than the conventional type diode. As a result, it is considered that the rectification effect of the fluidic diode can be improved by installing a bypass to the blunt body. Moreover, among the bypasses, taper cylindrical type with $\theta=5^{\circ}$ in which the pressure difference $\Delta p_{f}$ decreases and the flow rate in the positive direction increases, can be considered to be the most suitable type of bypass adopted within the scope of this study.

Figure 4 shows the velocity distribution in fluid diode in the case of forward flow. It can be seen in the figure that the air smoothly flows due to the vortices generated in the areas $T$ and $H$ while passing along the blunt body, and finally

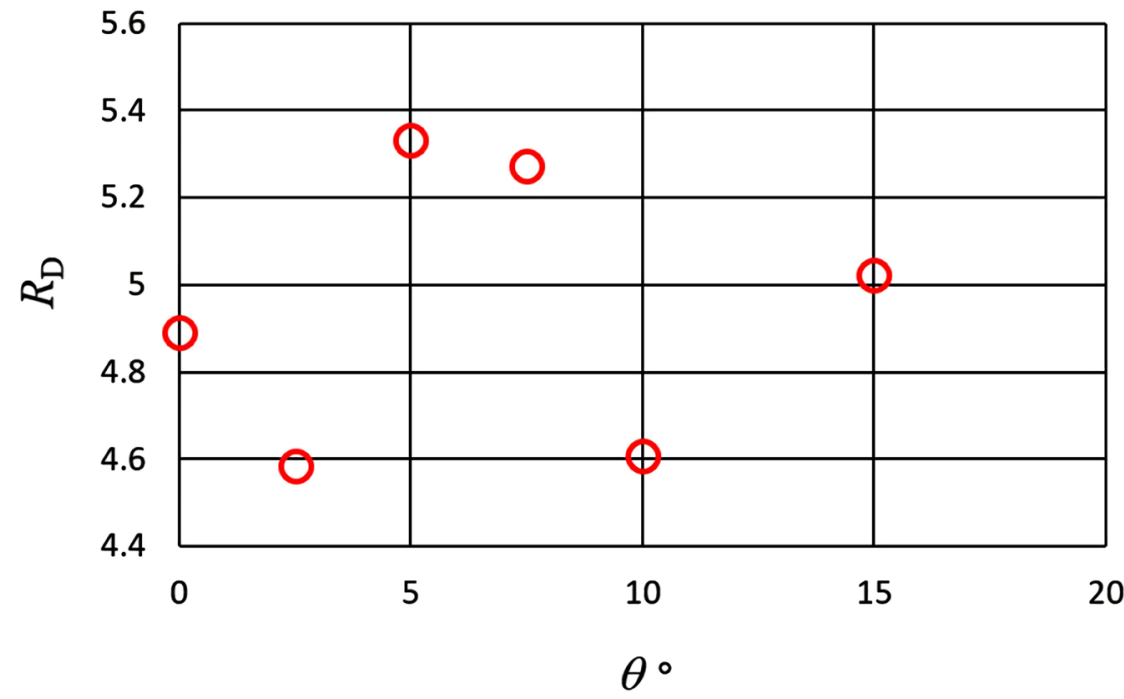

Figure 3. Effect of fluidic diode on pressure difference ratio (taper cylindrical type). 

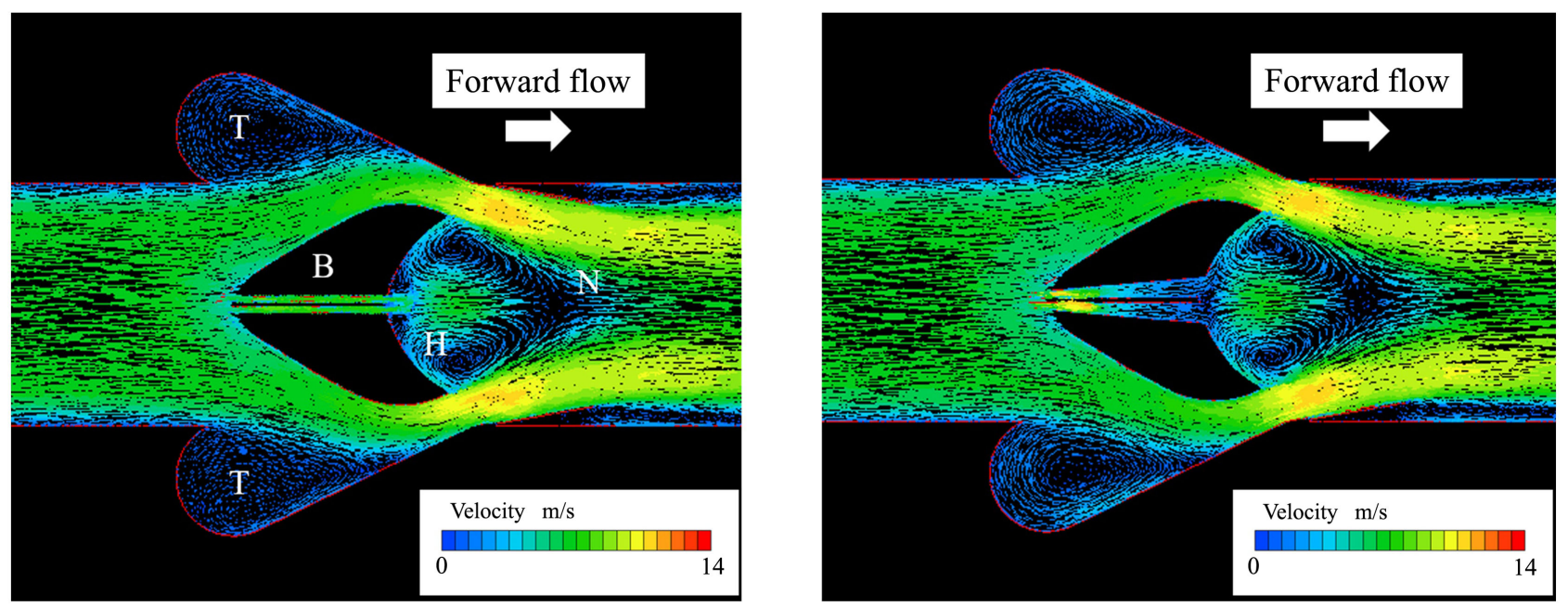

Figure 4. Velocity distribution in fluidic diode (forward flow). (A) Cylindrical type; (B) Taper cylindrical type $\left(\theta=5^{\circ}\right)$.

Table 1. Effect of bypass on rectification characteristics of the fluidic diode.

\begin{tabular}{ccccc}
\hline & \multirow{2}{*}{$\begin{array}{c}\text { Conventional } \\
\text { type diode }\end{array}$} & \multicolumn{3}{c}{ Fluidic diode with bypass hole } \\
\cline { 3 - 5 } & Straight type & Cylindrical type & Taper cylindrical type $\left(\theta=5^{\circ}\right)$ \\
\hline$\Delta p_{f}$ & 35.2 & 31.8 & 31.2 & 28.5 \\
$\Delta p_{r}$ & 142.9 & 149.5 & 152.7 & 151.8 \\
$R_{D}$ & 4.06 & 4.70 & 4.89 & 5.33 \\
\hline
\end{tabular}

passes through the area $N$. Further, in the case of reverse flow on shown in Figure 5, the airflow collides between the casing and the nozzle, and then collides in the areas $H$ and $T$. Thus, there is no collision found of high velocity in the forward flow. However, there are two collisions found at high velocity in the reverse flow and results in a large pressure difference, and that contributes to the rectification of the air flow.

In Figure 4(A), the vortex generated in a hollow $(H)$ at the back and the air flowing from the bypass collides with each other; leads to a separation. In Figure 4(B), the vortex generated at the depression and the airflow from the bypass flows smoothly without collision. As a result, the pressure distribution in Figure 6 shows that $\Delta p_{f}$ for the taper cylindrical type fluidic diode is low.

Figure 5 shows the velocity distribution in the fluidic diode in the case of reverse flow. It can be seen from Figure 5 that the taper cylindrical type fluidic diode has a larger bypass area than the cylindrical type; however, the flow does not change significantly. As a result, the pressure distribution in Figure 7 shows that taper cylindrical type has a higher rectification effect.

Hence, the effect of the fluidic diode on the flow rate $Q / Q$ and the efficiency $\eta$ of the twin-impulse turbine were estimated. The details of the turbine cascade can be found in Refs. [2] [3]. The turbine performance under the steady flow conditions in this case was evaluated by the input coefficient $C_{\mathrm{A}}$ against the flow coefficient $\phi$. The definitions of these parameters are as flows: 

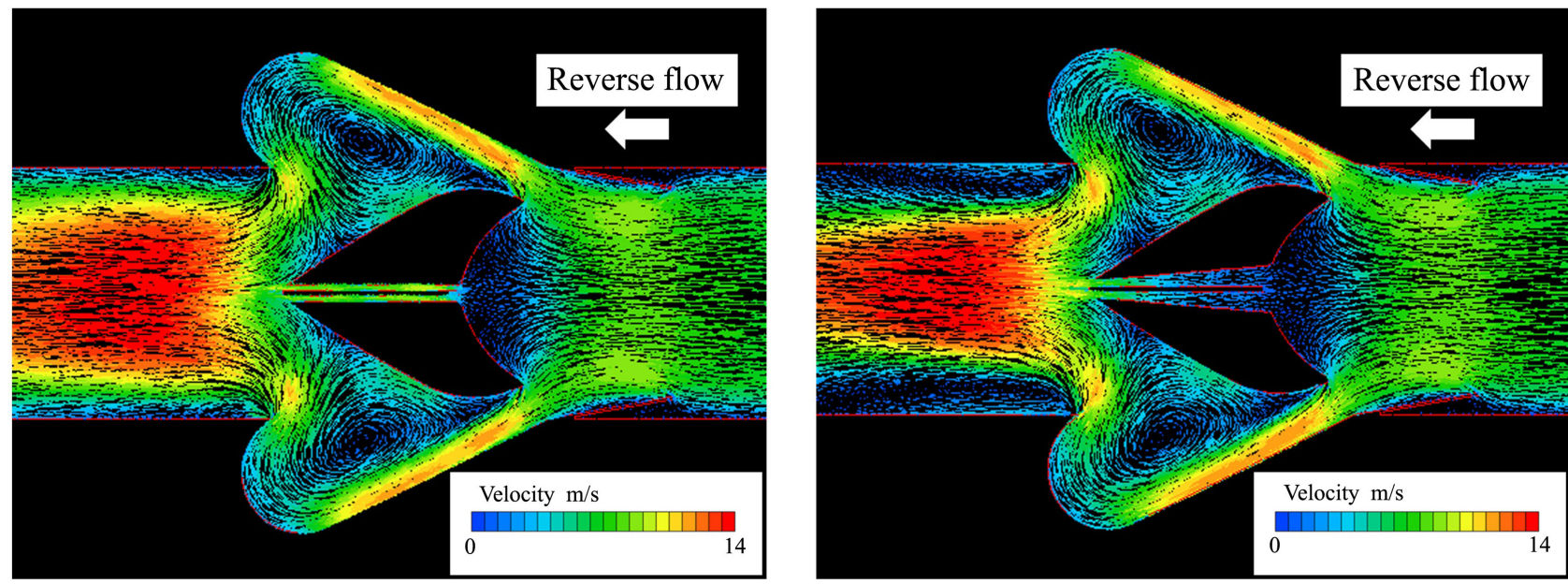

Figure 5. Velocity distribution in fluidic diode (reverse flow). (A) Cylindrical type; (B) Taper cylindrical type $\left(\theta=5^{\circ}\right)$.
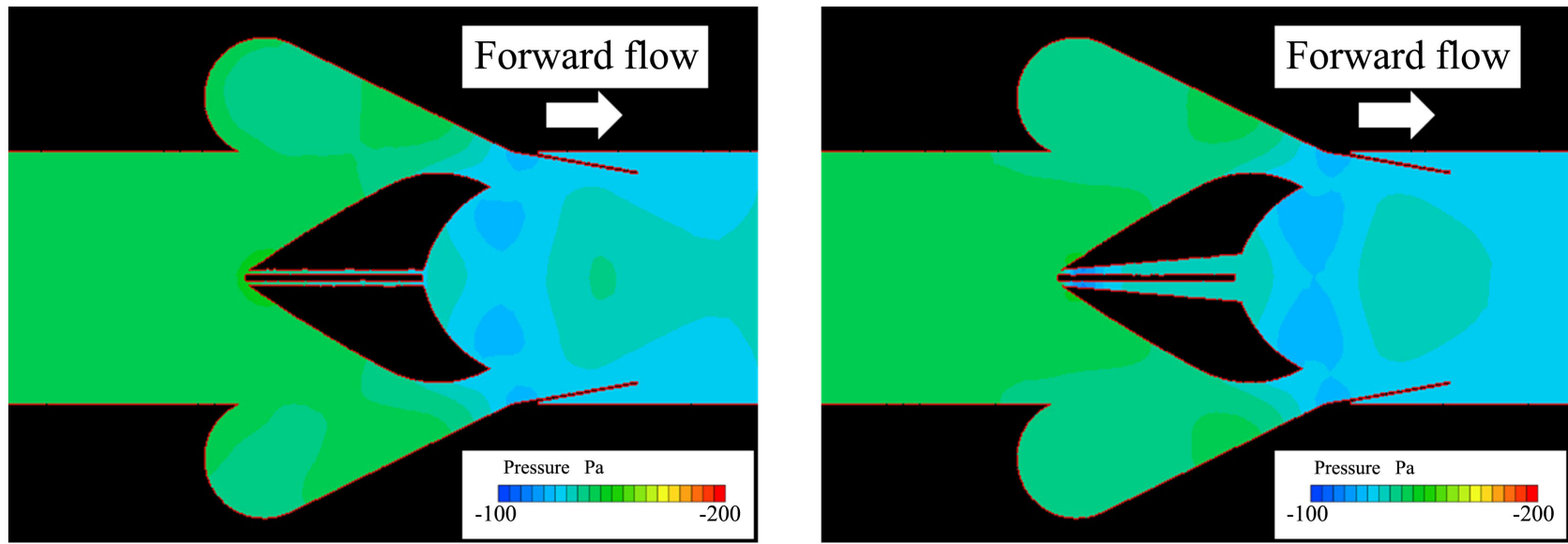

Figure 6. Pressure distribution in fluidic diode (forward flow). (A) Cylindrical type; (B) Taper cylindrical type $\left(\theta=5^{\circ}\right)$.
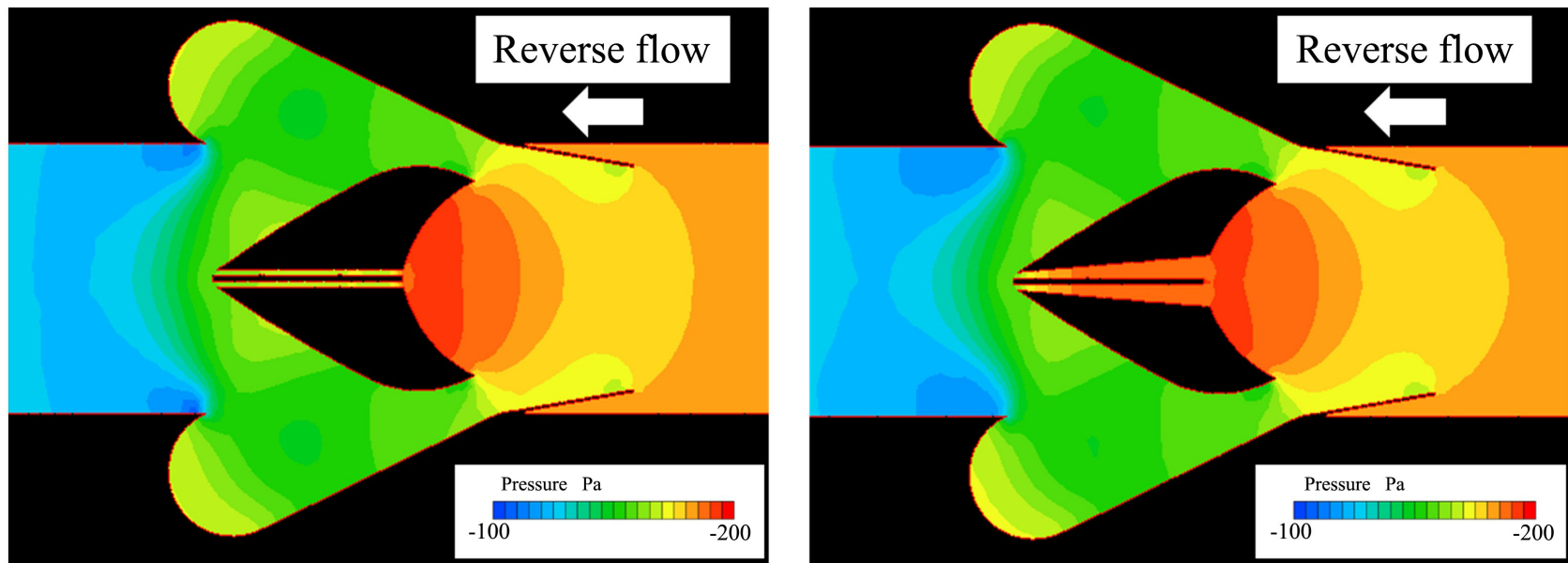

Figure 7. Pressure distribution in fluidic diode (reverse flow). (A) Cylindrical type; (B) Taper cylindrical type $\left(\theta=5^{\circ}\right)$.

$$
\begin{gathered}
C_{A}=\Delta p Q /\left\{\rho\left(v^{2}+u^{2}\right) A v / 2\right\}=\Delta p /\left\{\left(v^{2}+u^{2}\right) / 2\right\} \\
\phi=v / u
\end{gathered}
$$


where $A, r, u, v, \Delta p, \rho$ and $\omega$ denote the flow passage area $\left\{\pi D^{2}\left(1-v^{2}\right) / 4\right\}$, mean radius of the rotor $\{=D(1+V) / 4\}$, circumferential velocity at mean radius $(=r \omega)$, axial flow velocity $(=Q / A)$, pressure difference, density of air and angular speed of the rotor, respectively. The calculation was carried out by deriving a curve fit equation of flow coefficient $\phi$ from the experimental data shown in Figure 8.

In the estimation, the flow rates through the twin turbines were obtained by using the steady flow characteristics and solving these equations simultaneously.

$$
\begin{gathered}
Q=Q_{f}+Q_{r} \\
\Delta p=\Delta p_{f}=\Delta p_{r} \\
\eta=T_{o} \omega /(\Delta p Q) \\
\omega_{1}=\omega_{2}=\omega=\text { const. } \\
r=r_{f}=r_{r} \\
\phi_{f}=v_{f} / u_{f}=\left(Q_{f} / A\right) /(r \omega) \\
\phi_{r}=v_{r} / u_{r}=\left(Q_{f} / A\right) /(r \omega)
\end{gathered}
$$

where $Q$ and $T_{o}$ denote the flow rate through the turbine system and output torque. Subscripts $f$ and $r$ mean turbines in forward and reverse flows, respectively.

Figure 9 shows the effect of fluidic diode on the flow rate ratio in forward flow $Q_{f} Q$. As shown in the figure, the fluidic diode increases the flow rate in forward flow. It is considered that the installation of the fluidic diode decreases the flow rate in the reverse flow turbine, and increases the flow rate in the forward flow turbine.

Figure 10 shows the effect of fluidic diode on the efficiency of a twin impulse turbine in steady flow. From the figure, it can be seen that the efficiency increases in the range of low flow coefficient by installing the fluidic diode. However, when a fluidic diode is installed, the ratio of the pressure difference across the fluidic diode to the pressure difference before and after the turbine increases with

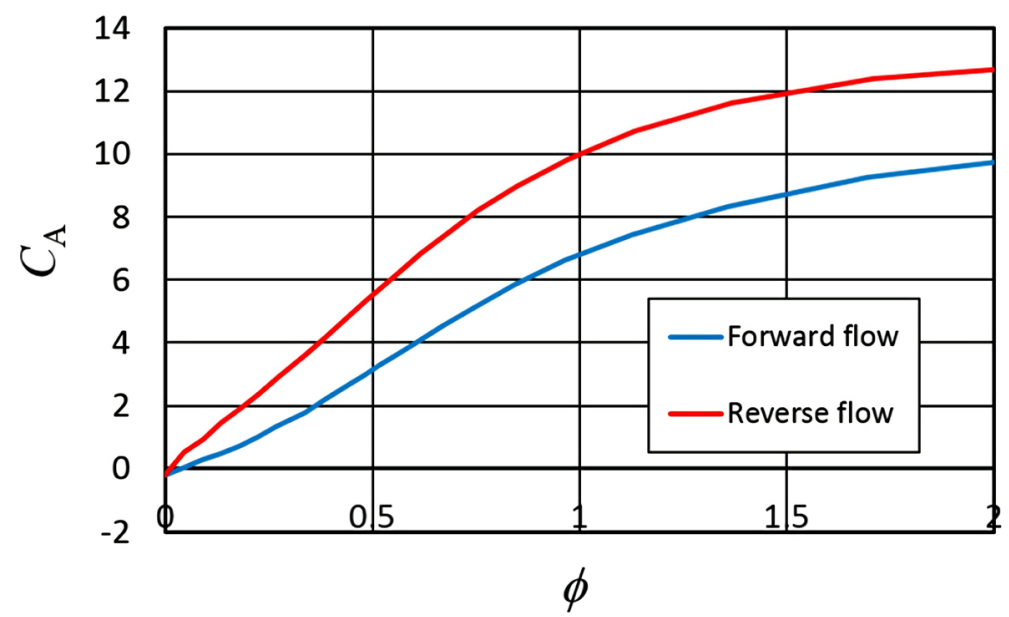

Figure 8. Input coefficient of impulse turbine for wave energy conversion [3]. 


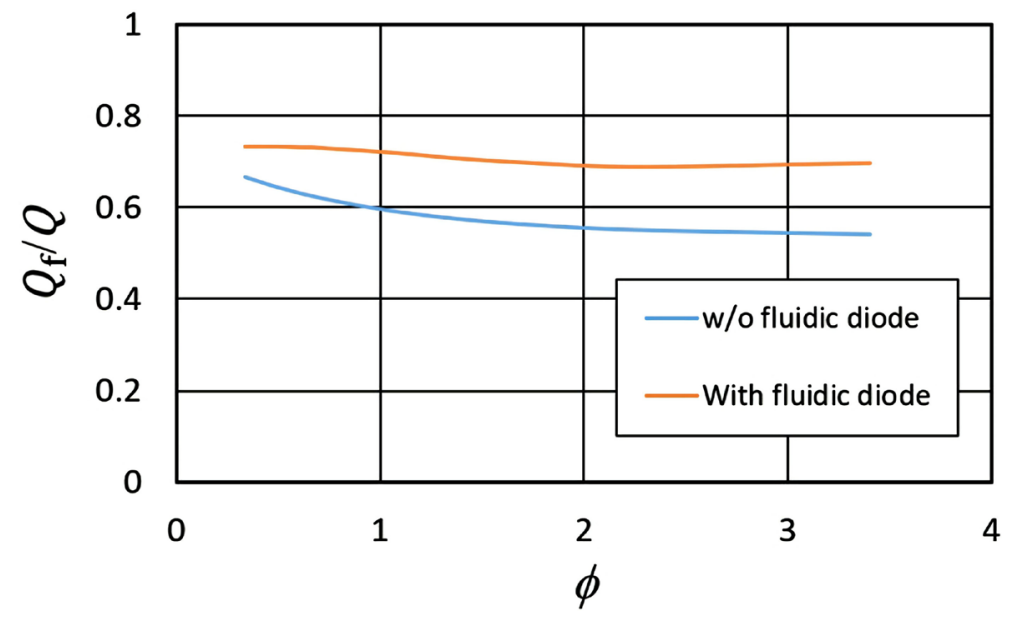

Figure 9. Flow rate ratio of forward flow (taper cylindrical type).

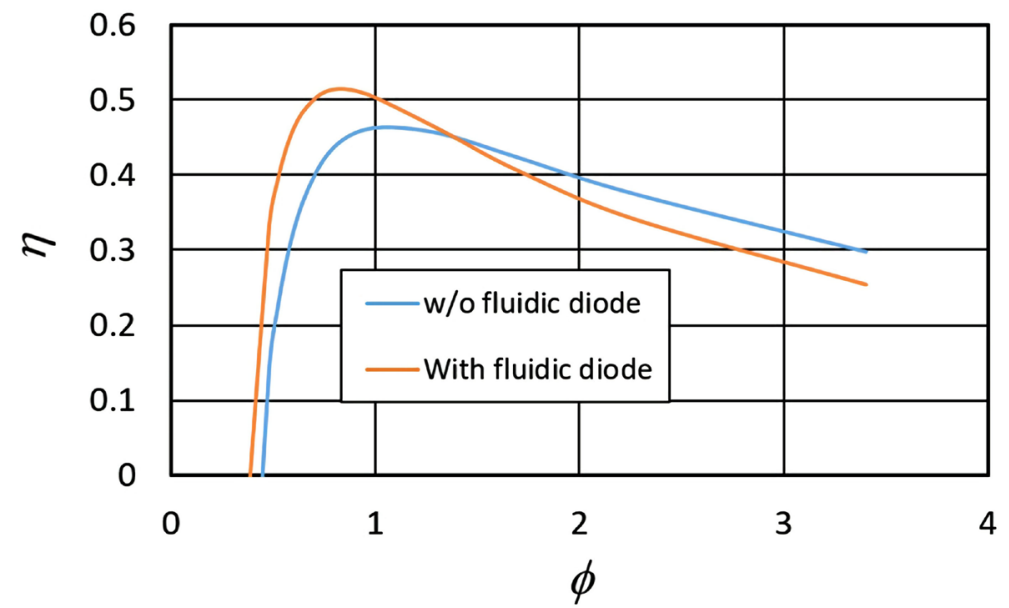

Figure 10. Turbine efficiency under steady flow condition (taper cylindrical type).

the flow coefficient $\phi$, and the efficiency is considered to be lower than that of a turbine without a diode. From this fact, it is considered that the installation of a fluidic diode in a twin impulse turbine to improve the commutation effect is effective only in the range of low flow coefficients.

\section{Conclusions}

In this study, in order to improve the performance of the fluidic diodes used in twin-impulse turbines for wave energy conversion, the effect of some bypass shapes to the fluidic diode on its rectification effect was investigated by a computational fluid dynamics (CFD) analysis. The obtained results are summarized below:

1) It was found that the installation of bypass with taper hole to the blunt body of the fluidic diode is effective to improve the rectification effect.

2) The preferred angle of taper cylindrical type bypass hole obtained is at $\theta=$ $5^{\circ}$ in the tested range.

3) From the results, the flow rate in the forward flow turbine found increases 
by installing a fluidic diode with taper cylindrical type bypass hole, and as it was expected, the turbine efficiency $\eta$ also increases.

\section{Conflicts of Interest}

The authors declare no conflicts of interest regarding the publication of this paper.

\section{References}

[1] Takao, M. and Setoguchi, T. (2012) Air Turbine for Wave Energy Conversion. International Journal of Rotating Machinery, 2012, Article ID: 717398. https://doi.org/10.1155/2012/717398

[2] Jayashankar, V., Anand, S., Geetha, T., Santhakumar, S., Kumar, V.J., Ravindran, M., Setoguchi, T., Takao, M., Toyota, K. and Nagata, S. (2009) A Twin Unidirectional Impulse Turbine Topology for OWC Based Wave Energy Plants. Renewable Energy, 34, 692-698. https://doi.org/10.1016/j.renene.2008.05.028

[3] Takao, M., Takami, A., Okuhara, S. and Setoguchi, T. (2011) A Twin Unidirectional Impulse Turbine for Wave Energy Conversion. Journal of Thermal Science, 20, 394-397. https://doi.org/10.1007/s11630-011-0486-1

[4] Okuhara, S., Takao, M., Sato, H., Takami, A. and Setoguchi, T. (2014) A Twin Unidirectional Impulse Turbine for Wave Energy Conversion-Effect of Fluidic Diode on the Performance. Open Journal of Fluid Dynamics, 4, 433-429.

https://doi.org/10.4236/ojfd.2014.45033 